\title{
Report
}

\section{MONTE CARLO SIMULATIONS AND BENCHMARK STUDIES AT CERN's ACCELERATOR CHAIN}

\author{
João Pedro SARAIVA and Markus BRUGGER
}

CERN EN/STI, CH-1211 Geneva 23, Switzerland

\begin{abstract}
Mixed particle and energy radiation fields present at the Large Hadron Collider (LHC) and its accelerator chain are responsible for failures on electronic devices located in the vicinity of the accelerator beam lines. These radiation effects on electronics and, more generally, the overall radiation damage issues have a direct impact on component and system lifetimes, as well as on maintenance requirements and radiation exposure to personnel who have to intervene and fix existing faults. The radiation environments and respective radiation damage issues along the CERN's accelerator chain were studied in the framework of the CERN Radiation to Electronics (R2E) project and are hereby presented. The important interplay between Monte Carlo simulations and radiation monitoring is also highlighted.
\end{abstract}

Keywords: CERN PS, CERN PS Booster, R2E project, Injector Chain, Radiation Environments, Dosimetry, Monte Carlo Calculations.

Presented at:

NSS 2015 - IEEE Nuclear Science Symposium \& Medical Imaging Conference

San Diego, USA

November, 2015 


\title{
Monte Carlo Simulations and Benchmark Studies at CERN's Accelerator Chain
}

\author{
João P. Saraiva and Markus Brugger, CERN EN/STI
}

\begin{abstract}
Mixed particle and energy radiation fields present at the Large Hadron Collider (LHC) and its accelerator chain are responsible for failures on electronic devices located in the vicinity of the accelerator beam lines. These radiation effects on electronics and, more generally, the overall radiation damage issues have a direct impact on component and system lifetimes, as well as on maintenance requirements and radiation exposure to personnel who have to intervene and fix existing faults. The radiation environments and respective radiation damage issues along the CERN's accelerator chain were studied in the framework of the CERN Radiation to Electronics (R2E) project and are hereby presented. The important interplay between Monte Carlo simulations and radiation monitoring is also highlighted.
\end{abstract}

\section{INTRODUCTION}

$\mathbf{M}$ IXED particle and energy radiation fields present at the Large Hadron Collider (LHC) and its accelerator chain are responsible for failures on electronic devices located in the vicinity of the accelerator beam lines. These radiation effects on electronics and, more generally, the overall radiation damage issues have a direct impact on component and system lifetimes, as well as on maintenance requirements and radiation exposure to personnel who have to intervene and fix existing faults.

In the framework of the CERN Radiation to Electronics (R2E) project [1], the radiation environments and respective radiation damage issues along the Injector Chain were studied during the last years. A detailed analysis of the dosimetry and beam monitoring available in the Injectors was carried out in parallel. Moreover, Monte Carlo calculations were performed to complement experimental data on the one side and for benchmark purposes on the other. Cross-checking all the collected information with the inventory of exposed equipment and systems [2], allowed us to evaluate possible medium or long-term radiation impacts in the Injectors and, if necessary, to implement mitigation actions as described later on.

The radiation environments and respective radiation damage issues along the CERN's accelerator chain were studied in the framework of the CERN Radiation to Electronics (R2E) project and are hereby presented. The dosimetry and beam monitoring systems used at CERN to assess radiation levels are also summarized in this paper. The important interplay between Monte Carlo simulations and radiation monitoring is also highlighted.

Manuscript received November 22, 2015.

João P. Saraiva and Markus Brugger are with the European Organization for Nuclear Research (CERN), Switzerland (e-mails: joao.pedro.saraiva@cern.ch, Markus.Brugger@cern.ch).

\section{Radiation to Electronics (R2E) Project}

The R2E project [1] started in 2007 to reduce the risk of electronic failures due to radiation and to increase the $\mathrm{R} 2 \mathrm{E}$ related mean time between failures (MTBF) of the LHC above 1 week, i.e. below $\sim 0.5$ beam dumps per inverse femtobarn $\left(\mathrm{fb}^{-1}\right)$ for nominal LHC beam conditions ${ }^{1}$. Mitigation actions implemented in the LHC during the last years decreased the number of beam dumps related to R2E failures to only few dumps per inverse femtobarn in 2012 and the expected evolution is to reach the required target level for LHC Run-2 (see Fig. 1).

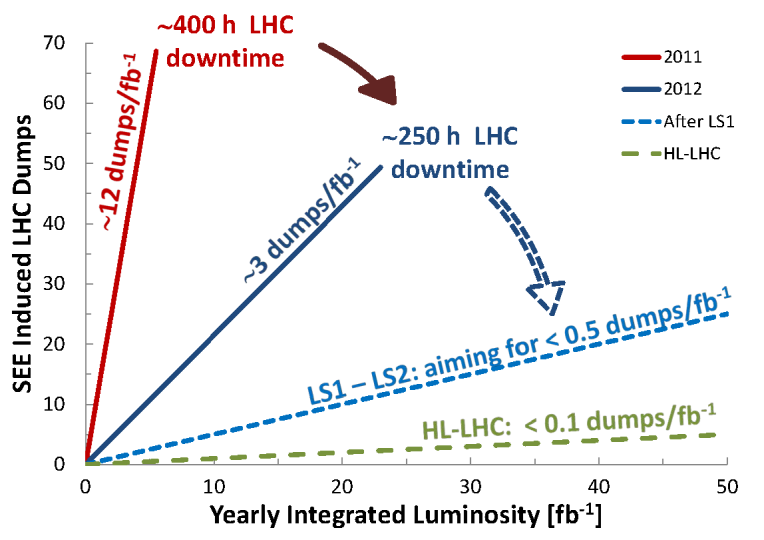

Fig. 1. R2E downtime over the last years and expected evolution after the first long shutdown (2013 to 2015).

In the next 10 years, the High Luminosity LHC (HL-LHC) upgrade project aims to attain around $250 \mathrm{fb}^{-1}$ per year (and more for ultimate upgrade performance) which can only be achieved increasing the peak luminosity by a factor of 5 compared to the nominal value and aiming to maximize overall machine availability. In the latter context, and as can be seen from Fig. 1, in order to fulfil the HL-LHC objective and to keep the R2E MTBF above 1 week, R2E related beam dumps will have to decrease even further, to less than 0.1 dumps/fb ${ }^{-1}$, ideally with no remaining R2E related premature dumps. Therefore, since 2012 additional R2E emphasis has also started to be put on the Injector Chain, which provides, among others, beam to the LHC experiments and in this way can contribute increasing the LHC downtime.

As shown in Fig. 2 several levels of information had to be collected and combined with Monte Carlo calculations to respond to the increasing demand from equipment owners

\footnotetext{
${ }^{1}$ Design LHC peak luminosity: $\sim 10^{34} \mathrm{~cm}^{-2} \mathrm{~s}^{-1}$ and expected yearly integrated luminosity of $\sim 50 \mathrm{fb}^{-1}$.
} 
to install electronic devices in the Injector Chain (e.g. for beam diagnostic, safety of individuals, etc.) and to protect or relocate the already existing devices in order to reduce radiation failures. These levels of information include:

- Layout of the different accelerators, transfer lines and experimental halls in the Injector Chain which corresponds to more than $18 \mathrm{~km}$ of tunnel length;

- Beam related topics such as beam intensities (for each machine) over the years, beam losses along the accelerators, trying to understand where and why they occur but also their expected evolution with respect to the current ongoing Injectors upgrade;

- Radiation monitoring carried out throughout the whole Injector Chain comprising prompt and residual dose measurements from the DGS-RP group, prompt dose and particle fluences measured by the EN-STI group with passive and active dosimeters, SRAM memories and PIN diodes.

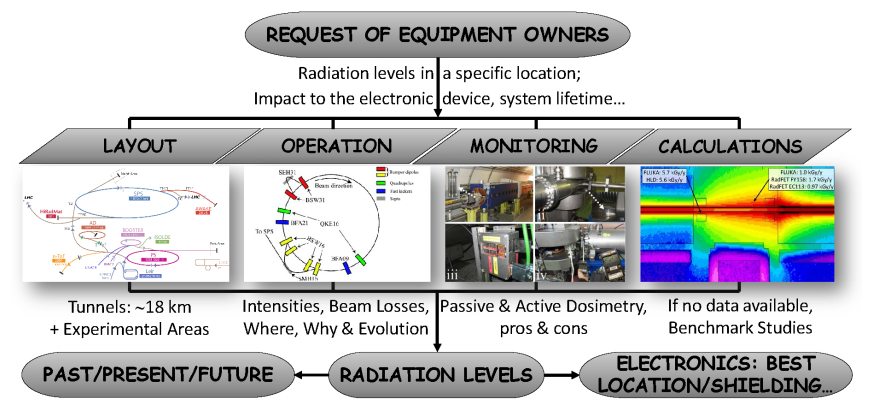

Fig. 2. Flow chart illustrating the different levels of information mastered in the framework of the R2E project in order to assess the radiation levels along the $18 \mathrm{~km}$ of Injector Chain (accelerators + transfer lines) and Experimental Areas.

\section{CERN'S INJECTOR CHAIN AND THE LIU PROJECT}

CERN's Injector Chain supplies particle beams to the LHC and a set of experimental areas as depicted in Fig. 3. Nowadays, the proton chain consists of one linear accelerator (LINAC2) and three synchrotrons, the PSB, PS and SPS, that successively increase the energy of the particle beam until injection into the LHC.

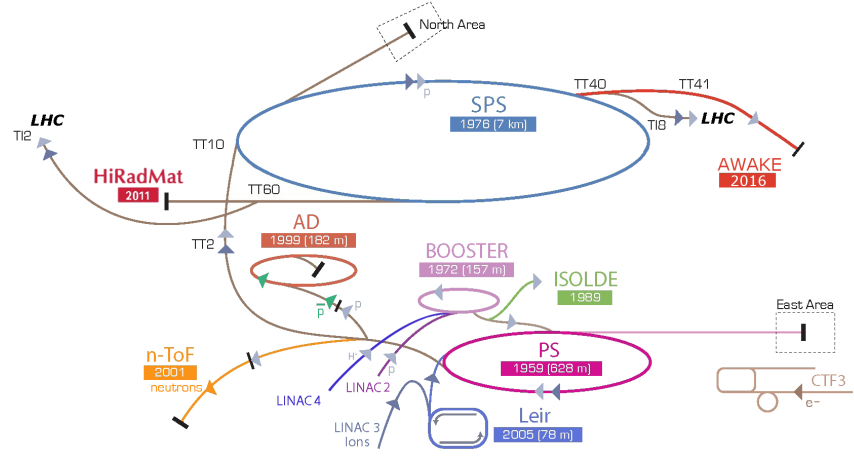

Fig. 3. CERN's Injector Chain.

Moreover, the ion injector chain comprises the LINAC 3 and the cooler and accumulator ring LEIR, also depicted in
Fig. 3, which feeds the PS with ion beams following, from there, the same route until the LHC.

To fulfil the ambitious objectives of the HL-LHC project for the next decade, of supplying high intensity and brightness beams to the LHC, the accelerator chain is undergoing a major improvement programme framed under the LHC Injectors Upgrade (LIU) project.

The main changes comprise the new Linac 4 which will replace the Linac 2, injecting $\mathrm{H}^{-}$ions of $160 \mathrm{MeV}$ in the PSB, thereby doubling its beam brightness [3]. For its part, the PSB extraction energy will be upgraded to a top energy of $2 \mathrm{GeV}$ reducing beam space-charge effects in the PS [4]. In the framework of the LIU project, several other upgrades were already implemented during the first long shutdown (LS1). As an example, in the PS, a longitudinal damper cavity based on the wideband frequency characteristics of Finemet ${ }^{\circledR}$ magnetic alloy [5] was installed in Straight Section 2 to fight against longitudinal coupled bunch instabilities and meet the high-brightness beam requirements of the LIU project. The shielding used to protect the amplifiers of the mentioned cavity was designed and implemented based on the Monte Carlo simulation presented below.

\section{RADIATION EFFECTS TO ELECTRONICS}

The Mixed radiation environments found along the CERN's accelerator chain affect exposed electronic devices through three different effects (see Fig. 4):

- Single Event Effects (SEE): stochastic effects due to the energy deposition of a single incident particle traversing the semiconductor structure. Electron-hole pairs generated along the path of the ionizing particle give rise to a transient current pulse in the sensitive volume of the device resulting in its failure, for instance, modifying the state of a memory bit (Single Event Upset) or leading even to destructive failures. Its likelihood of occurrence is correlated with the integrated hadron fluence ${ }^{2}$;

- Ionizing Effects: cumulative effect related to the charge build-up (trapped holes) in silica insulator $\left(\mathrm{SiO}_{2}\right)$ when exposed to ionizing radiation and resulting in gradual parameter degradation of electronic components over time, e.g. the threshold voltage drift in a MOS-structure. Ionizing effects are evaluated by the cumulated Total Ionizing Dose (TID);

- Non-Ionizing Effects: cumulative effect linked to the increased number of atomic displacements in the semiconductor lattice. The energy of the incoming particles is imparted to recoil nuclei creating lattice defects, increasing the number of recombination centres and resulting in the degradation of the semiconductor behaviour. The representative quantity for displacement damages is the integrated $1 \mathrm{MeV}$ equivalent neutron fluence.

\footnotetext{
${ }^{2}$ In mixed radiation environments without ions as primary particles (as opposed to the space environment) it is the secondary particles emerging a nuclear reaction which are the actual source of SEEs. In a simplified approximation one can say that above $10-20 \mathrm{MeV}$ the reaction cross section is constant (only valid for SEUs and silicon as contributing target material), thus the total fluence above this threshold is a measure of the SEE failure probability
} 


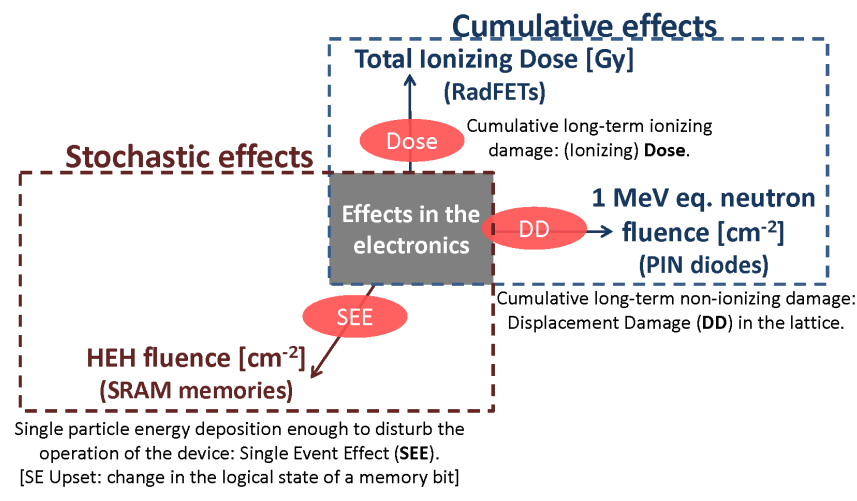

Fig. 4. Main radiation effects on electronic devices, their representative quantities and respective monitoring devices.

\section{DOSIMETRY, RADIATION SURVEYS \& BEAM MONITORING}

A key source of information to assess the radiation levels in the Injectors relies on dosimetry and radiation surveys performed throughout the years. Fig. 5 shows few examples of detectors used along the accelerator chain for prompt and residual dose measurements.
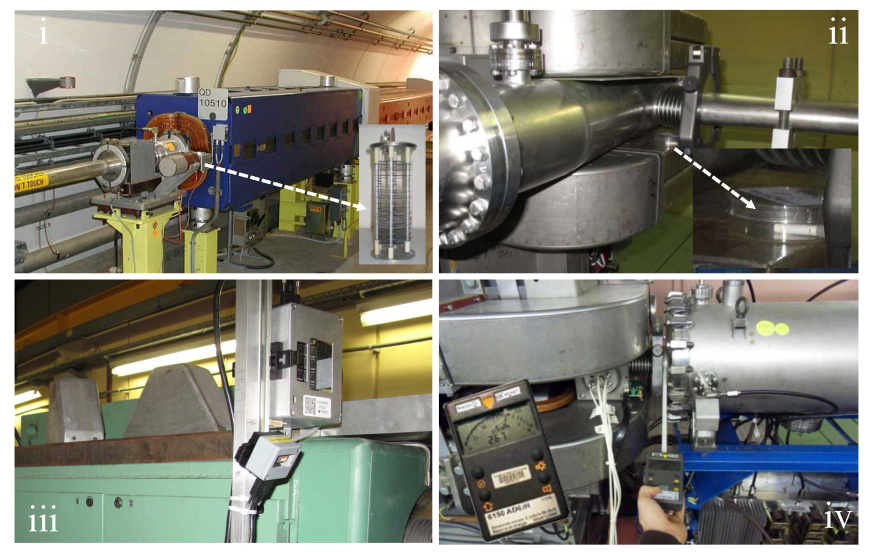

Fig. 5. Radiation monitoring systems used along the Injector Chain Beam Loss Monitor (BLM) close to the beam line of the SPS (i); RadioPhotoLuminescent Glass Dosimeters (RPL) installed just below the PS vacuum chamber (ii); Radiation Monitoring (RadMon) near a bending magnet of the PSB (iii); residual dose rate survey performed with a Geiger-Müller $(\mathrm{AD} 6 / \mathrm{H})$ at $40 \mathrm{~cm}$ of distance to the PS vacuum chamber (iv).

\section{A. Passive dosimetry}

Most of the passive dosimetry performed at CERN is based on two types of dosimeters, Radio-PhotoLuminescent Glass Dosimeters (RPLGD or simply RPL) (see Fig. 5 (ii)) and, more recently, Radiation sensitive Field Effect Transistors (RadFET).

The RPL dosimeters are passive solid-state dosimeters, based on the photoluminescence phenomenon of a glass compound, the silver activated metaphosphate glass. Without going into details, when exposed to ionizing radiation, electron-hole pairs are created from the metaphosphate substrate (empirical formula: $\mathrm{PO}_{3}^{-}$). Both, electrons and holes then combine with $\mathrm{Ag}^{+}$ions, creating so called stable colour centres, $\mathrm{Ag}$ and $\mathrm{Ag}^{2+}$ respectively. When excited with a pulsed UV laser, these colour centres emit visible light which intensity is proportional to the number of colour centres and therefore to the absorbed dose.

The RadFET dosimeter is a pMOSFET sensitive to ionizing radiation owing to the build-up of radiation induced positive charges in the $\mathrm{SiO}_{2}$ gate dielectric. The presence of these trapped holes results in the shift of the threshold voltage, which can in turn be converted to the total ionizing dose (TID) imparted to the transistor.

\section{B. Active dosimetry}

1) RadMon System: Installed in 2013/2014 during the LS1 in the PSB, PS and SPS, the RadMon system (see Fig. 5 (iii)) brought huge benefits to the dosimetry performed in the Injector Chain. Firstly, it provides real-time measurements of TID which affects almost all kind of materials in radioactive environments. Moreover, and since electronic devices are usually the most vulnerable in such environments, the RadMon system also allows real-time measurements of two particle fluences, the already mentioned high energy hadron (HEH) and $1 \mathrm{MeV}$ equivalent neutron ( $1 \mathrm{MeV}$ eq. n.) fluences. On top of this, these measurements can be obtained at any point of interest, close to the accelerator beam line, near the tunnel walls, etc. The versatility of the CERN-made RadMons is achieved by using two monitoring devices, a main board and a deported unit which can be placed up to 200 meters from the main device.

2) Beam Loss Monitors (BLM): The Beam Loss Monitor (BLM) system currently used in the PSB and PS is based on the Aluminium Cathode Electron Multiplier (ACEM) detector (Photomultiplier tube of enhanced sensitivity to ionizing radiation achieved by replacing the photocathode by an aluminium foil). However, this system, installed in 1975 and 1983 in the PS and PSB respectively, can only be used for qualitative measurements of the beam losses. Due to several drawbacks, such as saturation in critical areas, the ACEM detectors are of limited use even to establish beam loss patterns along the machine, useful for Monte Carlo simulations. The upgrade of the BLM system in the PSB has been launched during the LS1 while in the PS is currently planned for LS2. In the SPS, the ring BLM system consists of ionization chambers installed along the machine each 32 meters (see Fig. 5 (i)). They can be used for prompt dose measurements and when the beam is off, in high gain mode, for residual dose measurements.

\section{Radiation Survey}

Despite the fact that residual dose rate measurements are dependent upon induced activation of the accelerator surroundings, the residual radiation remaining after beam-off provides a footprint of beam losses and thus of prompt radiation along the machine when the beam was on. Several radiation monitoring surveys are performed every year by the CERN RP Group. Fig. 5 (iv) shows an AD6 detector used during a radiation survey in the PS accelerator. 


\section{RADIATION ENVIRONMENTS IN THE INJECTOR CHAIN}

Radiation fields in the LHC's Injector Chain were assessed on the basis of two main levels of information, on the one hand, the extended analysis of beam and radiation monitoring and on the other, FLUKA Monte Carlo calculations [6], [7] performed for all the main accelerators of the CERN's Injector Chain, the Linac 4, PSB, PS and SPS. Fig. 6 gives an overview of radiation levels ${ }^{3}$ that can be found in the main Injectors near the respective beam lines and tunnel walls, comparing them to the levels in the LHC and other radiation environments.

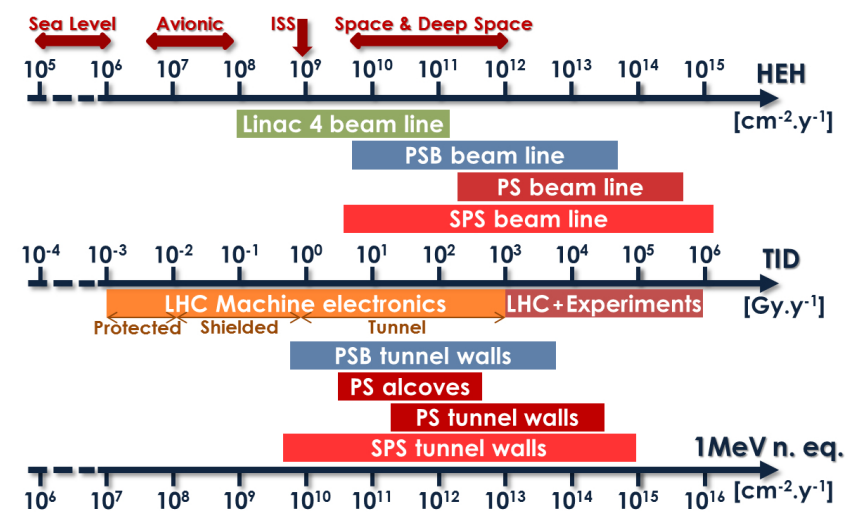

Fig. 6. Overview of the typical radiation levels found along the CERN's accelerator complex, expressed by the representative quantities of radiation damage on electronics, namely the yearly TID, $\mathrm{HEH}$ and $1 \mathrm{MeV}$ equivalent neutron fluences.

From Fig. 6 one can see that prompt dose levels can reach the MGy level near the vacuum chamber of the SPS accelerator in only one operational year. As depicted in the figure, around one order of magnitude separates the TID near the vacuum chamber and the respective tunnel walls, a rule of thumb based on TID measurements and supported by calculations [8].

\section{FLUKA Monte Carlo Simulations}

\section{A. CERN PS}

As previously mentioned, solid-state amplifiers for a wideband kicker cavity $(0.5-5.5 \mathrm{MHz})$ were installed during the LS1 in the PS accelerator (straight section 02, SS02). In order to meet the amplifier specifications they were located on the floor at only one meter of distance to the vacuum chamber.

According to the radiation levels in SS02 and to radiation tests carried out at J-PARC Main Ring, the amplifiers would not survive more than 1 year at this location. Since the electronics could not be moved away from the beam line and in order to increase the amplifier lifetime expectancy by a factor of at least 10, a cast iron shielding, designed based on the present FLUKA calculation, was installed to protect the amplifiers.

\footnotetext{
${ }^{3}$ Typical values measured over the last years along the accelerator chain, complemented with FLUKA simulations.
}
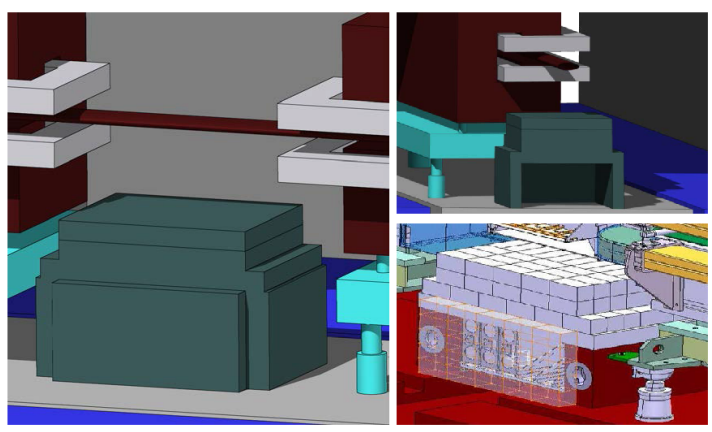

Fig. 7. FLUKA and integration geometries of $40 \mathrm{~cm}$ of cast iron shielding installed in SS02 during the LS1 for the RF wideband kicker cavity.

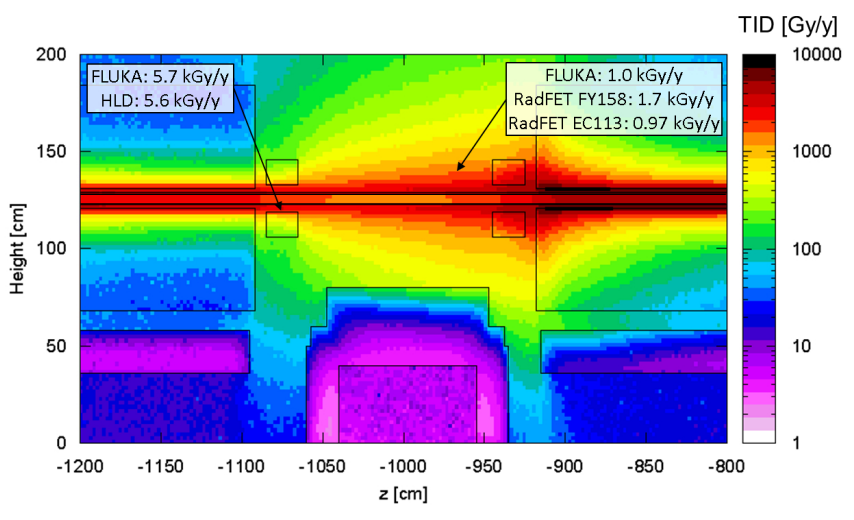

Fig. 8. 2D map of the yearly TID [Gy. $\mathrm{y}^{-1}$ ] in $\mathrm{SS} 02$ with $40 \mathrm{~cm}$ of cast iron shielding protecting the amplifiers. A comparison between calculations and prompt dose measurements is also presented.

Fig. 7 shows the implemented geometry of the shielding while Fig. 8 provides the results of the calculation showing also a comparison with prompt dose measurements performed during the 2012 operation period.

\section{B. PS Booster}

The recurrent lack of correlation between induced activation and prompt dose measurements found in the past in the accelerator PS Booster led to the launching of a FLUKA simulation of the whole machine in order to carry out, for this accelerator, the first benchmark study between calculations and passive dosimeters. The motivation for this study arises also from the fact that, up until now, no information was available at all with regard to the radiation levels near the PSB tunnel walls. Therefore, in 2014 a first batch of 60 RPL dosimeters were installed near the vacuum chamber and in both inner and outer tunnel walls of periods 08 and 10 (see Fig. 9). Fig. 10 shows the implemented geometry (which for this study included only bending and quadrupole magnets).

The results of the first batch of dosimeters were compared with calculated values (see Fig. 11) and the ratios between TID near the beam line and the respective tunnel walls were also established (see Fig. 12). To confirm the experimental results, a second batch of 60 dosimeters is currently installed and being irradiated in the PSB until the end of 2015 . 

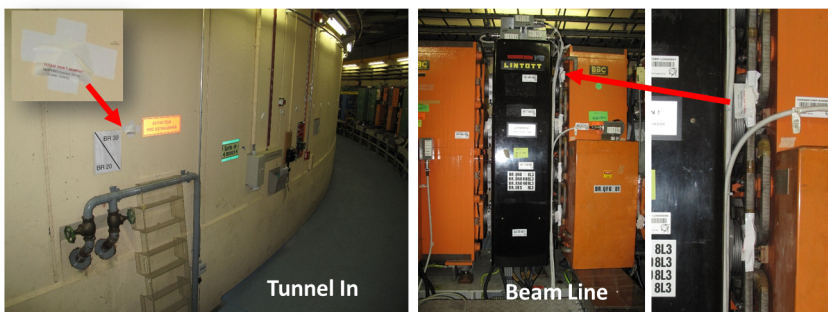

Fig. 9. Location of the passive dosimeters near the PSB beam line and the inner tunnel wall.

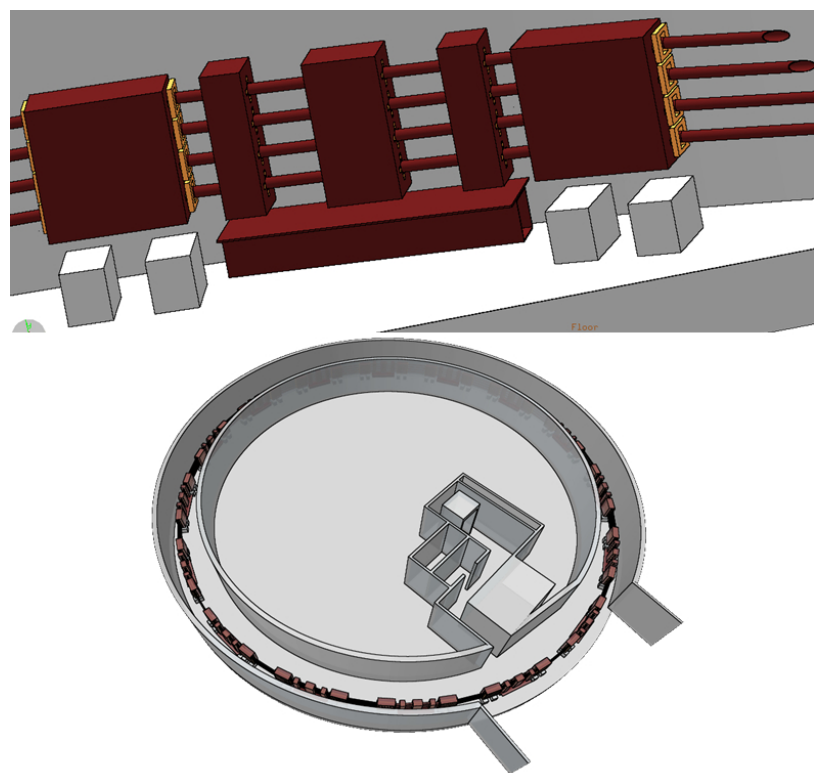

Fig. 10. On the bottom, implemented geometry of the whole PSB accelerator. On the top, one period in more detail.

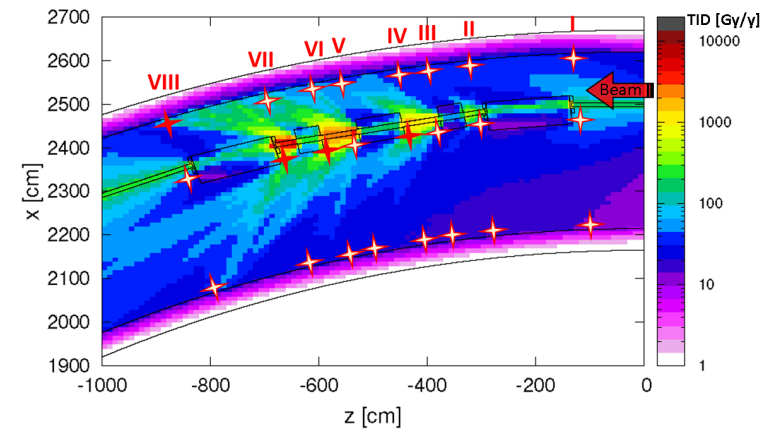

\begin{tabular}{lcccccccc}
\hline FLUKA/meas. & BM-1U & BM-1D & QF-1D & QD-U & QD-D & QF-2U & BM-2U & BM-2D \\
\hline Beam Line & 3,3 & 0,9 & 3,9 & 20,8 & 1,6 & 6,9 & 10,9 & 2,4 \\
Tunnel_In & 3,2 & 1,7 & 1,4 & 1,1 & 1,1 & 1,3 & 2,0 & 4,8 \\
Tunnel_Out & 2,5 & 1,8 & 1,3 & 2,5 & 3,0 & 2,8 & 3,6 & 7,8 \\
\hline
\end{tabular}

Fig. 11. Ratios between the TID calculated and measured along periods 08 of the PSB. Elements like multipole magnets were not implemented in this geometry, resulting in few discrepancies, specially near the beam line.

For this study, the full geometry of the accelerator was established. However, in the future more elements like multipole magnets will have to be implemented in the geometry in order to overcome some discrepancies found between measured and calculated values, specially near the vacuum chambers (see Fig. 11). Assumptions taken to perform the simulation may also have contributed to the mentioned discrepancies and to the overall uncertainties, namely:

- the loss profile of primary particles set in the calculation that is based on measurements from the residual dose rate survey of December 2014. Unfortunately, magnet realignments were performed in the PSB two months before, impacting directly on the beam loss pattern along the machine;

- the cooling time of the mentioned survey, of only 31 hours, which results in a high dependency on the behaviour of the accelerator during the last days of operation (no other survey of higher cooling time was performed in the PSB during this year-end technical stop);

- establishing the correspondence between the location of the dosimeters in the geometry used for the simulation and their real locations in the accelerator. The error arising from this assumption is higher at places with strong dose gradients, such as near the accelerator beam line.

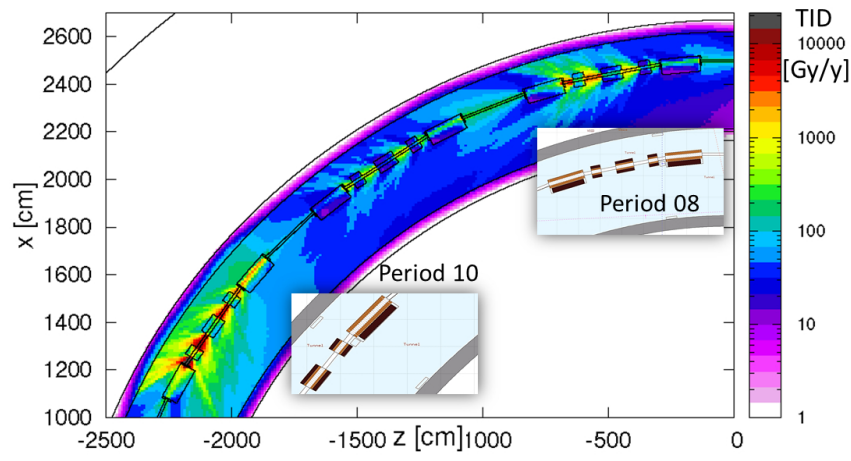

\begin{tabular}{lll} 
Ratios from FLUKA calculation: & & Ratios from measurements: \\
\cline { 1 - 1 } Bline $/$ Tin $=11.4 \pm 6$ & & Bline $/$ Tin $=9.2 \pm 3.3$ \\
Bline $/$ Tout $=4.9 \pm 2$ & Bline $/$ Tout $=4.8 \pm 1.7$ \\
Tout $/$ Tin $=2.3 \pm 0.2$ & Tout $/$ Tin $=2.0 \pm 0.9$
\end{tabular}

Fig. 12. Ratios between the TID close to the vacuum chambers and near the respective tunnel walls. Information used to optimize the location of electronic devices in the accelerator

The ratios between the TID near the vacuum chambers of the PSB and its tunnel walls are a valuable information for equipment owners, allowing them optimizing the location of their electronic devices, contributing increasing system lifetimes and reducing maintenance costs. This study showed that one can expect a TID reduction factor up to one order of magnitude between the PSB beam line and the respective inner tunnel wall, while the outer tunnel side presents, since closer to the beam line, twice the TID than the inner side (see Fig. 12).

\section{CONCLUSION}

The R2E project has been working during the last years to avoid electronics failures caused by radiation in the LHC. 
Since 2012, a special attention was paid to the LHC's Injector Chain with the intent of further reducing the global downtime, due to radiation-induced failures on electronics, to the lower possible level. In this paper, an overview of the radiation and beam monitoring systems available along the accelerator chain was shown. The radiation environments found in the different accelerators where also presented. The high radiation levels along the accelerator chain clearly show that the location of electronic devices must be chosen carefully in order to minimize the contribution of the radiation-induced failures to the overall accelerator downtime. Two benchmark studies between prompt dose measurements and FLUKA Monte-Carlo calculations were also presented for the PS and PS Booster accelerators, emphasizing the powerful interplay between calculations and radiation monitoring. Based on the results of the PS simulation, a shielding of $40 \mathrm{~cm}$ of cast iron was installed around amplifiers located in the straight section 02 in order to increase their lifetime expectancy by a factor of 10 . With regard to the PSB simulation, the radiation levels near the tunnel walls were calculated for the first time and compared to measurements, showing a factor of 10 between the TID near the beam line and the inner tunnel wall of the accelerator and a factor of 5 between the same beam line and the outer tunnel wall.

\section{ACKNOWLEDGMENT}

The authors would like to acknowledge all the suggestions and information provided by colleagues from many groups at CERN, namely the EN/STI-CV-EL, DGS/RP, BE/ABP-OPBI-RF groups.

\section{REFERENCES}

[1] The R2E Project: http://r2e.web.cern.ch/R2E.

[2] http://r2e-injectors.web.cern.ch

[3] Gerigk F. and Vretenar M. (Editors), "Linac 4 Technical Design Report", CERN-AB-2006-084 ABP/RF (2006).

[4] Gilardoni S. et al., "The PS Upgrade Programme: Recent Advances", Proceedings of IPAC2013 (2013).

[5] Persichelli S. et al., "Impedance Studies for the PS Finemet ${ }^{\circledR}$ loaded longitudinal damper", Proceedings of IPAC2014 (2014).

[6] A. Ferrari, P. R. Sala, A. Fassò and J. Ranft, "FLUKA: a multi-particle transport code”, CERN-2005-10, INFN/TC_05/11, SLAC-R-773 (2005).

[7] Battistoni G., Muraro S., Sala P. et al., "The FLUKA code: description and benchmarking, Proceedings of the Hadronic Shower Simulation Workshop 2006", Fermilab (2006).

[8] Saraiva J. and Brugger M., Radiation Environments and their Impact at the CERN's Injector Chain, CERN-ATS-Note-2016 (2016). 